\title{
Classifiche e rape
}

\section{Rankings and turnips}

Giovanni Lodi

Dipartimento di Scienze Biomediche, Chirurgiche e Odontoiatriche

Università degli Studi di Milano

via Beldiletto 1/3 Milano 20142

Italia

giovanni.lodi@unimi.it

tel. +390250319021

fax. +390250319041

In occasione del recente congresso che ha riunito i docenti di discipline odontostomatologiche ho voluto verificare lo stato di salute della ricerca odontoiatrica in Italia. Per farlo, ho utilizzato lo SCImago Journal \& Country Rank, uno strumento che permette di confrontare la produzione scientifica di riviste, settori disciplinari e nazioni.

Nelle classifiche che si basano sul numero di articoli pubblicati e citazioni ricevute, la ricerca odontoiatrica del nostro paese è al settimo posto, dietro giganti come Stati Uniti, Giappone, Regno Unito, Brasile, Germania e India. Mica male. Anche perché, se invece che basarci sulla quantità, proviamo a misurare la qualità, contando il numero medio di citazioni ricevute dagli articoli, allora facciamo un grande balzo in avanti, fino a precedere tutte queste nazioni. Gli odontoiatri italiani pubblicano molto e pubblicano bene. Risultati simili a quelli della ricerca italiana nel suo insieme, che si piazza all'ottavo posto per tutti gli indicatori a cui abbiamo appena accennato.

Qualcuno a questo punto potrà pensare che allora le continue lamentazioni di ricercatori e scienziati sulla poca attenzione e lo scarso sostegno dato dal nostro paese alla ricerca siano ingiustificate. E' vero l'esatto contrario. Continuate a leggere prego.

Qualche tempo fa il governo del Regno Unito ha reso disponibile un documento che non si limita a confrontare i numeri assoluti dei diversi paesi, ma ne misura la produttività, mettendo in relazione i risultati della ricerca con ciò che si è investito, sia in termini di economie che di personale (International Comparative Performance of the UK Research Base). Ebbene, l'Italia è sul podio insieme a Regno Unito e Canada nella classifica del numero di articoli e citazioni per milione di dollari investiti, e raggiunge il primo posto assoluto, staccando tutti gli altri paesi, quando si considera il rapporto tra prodotti della ricerca e numero di ricercatori impiegati.

Buone notizie? Non saprei. Queste classifiche ci dicono che i ricercatori italiani sono bravi, molto bravi, e che continuano a fare un ottimo lavoro nonostante l'intero sistema università (personale, studenti, fondi) si sia ridotto in pochi anni del $20 \%$. Avete letto bene, un quinto dell'università italiana è andato in fumo (questa volta la fonte è un rapporto della Fondazione Res pubblicato quest'anno). Cavare sangue dalle rape, ecco la missione della ricerca italiana. Difficile che duri.

Buona lettura 